\title{
Sagittal distraction of the sternum in a juvenile male with severe short rib syndrome and thoracic insufficiency syndrome - a case report
}

\begin{abstract}
Background: This report describes a case of thoracic insufficiency/short rib syndrome treated with sagittal distraction of the sternum in a juvenile male.

Methods: An acute increase in the thoracic cage volume was achieved in an 8-yearold male with thoracic insufficiency syndrome by sternum splitting and distraction of 3 centimeters. He had previously been treated with bilateral opening wedge and thoracic vertical expandable prosthetic titanium rib instruments. At 8 years, his respiration was rapidly deteriorating, and posterior revision surgery with resection of the posterior wall at this stage had no further positive influence on his respiratory function. Two years later, a second distraction of the sternum and acute distraction were performed as part of the thoracic expansion procedure.

Results:The procedure resulted in an increase in his respiratory functions, and his pulmonary artery parameters decreased to normal values. His clinical condition improved, and after surgery, he could ride a bicycle to school and even participate in sports, such as cross-country skiing and soccer.

Conclusion: Short rib syndrome presenting with respiratory failure in a neonate is potentially fatal without surgical intervention to expand the thoracic cage. Our patient had severe thoracic insufficiency syndrome and experienced clinical improvement from the procedure. Sternum distraction has been previously used in neonates, but to the best of our knowledge, this is the first distraction of the sternum addressing short rib syndrome in a juvenile.
\end{abstract}

Keywords: Short rib syndrome, Early onset scoliosis, Sternal distraction, Scoliosis, Thoracic insufficiency syndrome
Volume 9 Issue I - 2017

\author{
Thomas Johan Kibsgård, ${ }^{1,2}$ Rolf Riise' \\ 'Division of Orthopaedic Surgery, Oslo University Hospital, \\ Norway \\ ${ }^{2}$ University of Oslo, Norway
}

Correspondence: Thomas Kibsgard, P.b. 4950, 0424 Oslo, Norway Email rrsiise@ous.hf.no

Received: April 09, 2017| Published: September 20, 2017

\section{Abbreviations}

SRS, Short Rib Syndrome; TIS, Thoracic Insufficiency Syndrome; VEPTR, Vertical Expandable Prosthetic Titanium Rib; FVC, Forced Vital Capacity; FEV, Forced Expiratory Volume

\section{Introduction}

Short rib syndrome (SRS) with severe clinical respiratory impairment is a rare condition. The physiological development of the lungs is jeopardized due to a small thoracic cage and volume. ${ }^{1-3}$ In severe conditions, thoracic insufficiency syndrome (TIS) will ultimately develop. ${ }^{4}$ The literature contains several suggestions for treatment of SRS. ${ }^{5-8}$ but the outcomes are divergent, with reports of early deaths despite surgical interventions. The most commonly used surgical technique is a vertical expandable prosthetic titanium rib (VEPTR, DePuySynthes, Switzerland), but a limitation of this treatment is that the posterior and lateral thoracic cage tends to auto-fuse after long-term use. Several articles in the literature refer to rib and sternum procedures that increase the thoracic volume. ${ }^{5,8-11}$ and a sternum osteotomy can be an option in extreme cases if other procedures have been attempted. To the best of our knowledge, all references in the literature regarding this procedure involve populations of siblings; therefore, we aim to describe a case in which sagittal distraction of the sternum was used successfully in a juvenile male.

\section{Case presentation}

\section{Pre-operative medical history}

The patient was born with severe omphalocele and short ribs, but in contrast to Jeune syndrome, the patient also had fused ribs on the left side between ribs 3 and 6 . The patient showed immediate signs of respiratory distress following birth and required intubation shortly after birth (Apgar 7/10). Within the first weeks, he underwent several surgeries before his omphalocele was successfully treated. During this period, he was intermittently treated with a respirator, but at the age of four months, he was respirator-dependent and underwent a tracheostomy (Figure 1). At the age of 13 months, he was referred to the spinal unit. X-ray examination revealed both short ribs and fused ribs in the left hemi-thorax (Figure 2a). At 15 and 18 months, he underwent staged opening wedges (thoracic expansion procedures) with VEPTR instrumentation bilaterally (Figure 2b). Initially, after these procedures, his respiratory function was greatly improved; at the age 28 months, he was finally weaned off the respirator, and his tracheostomy was closed. The patient underwent standard lengthening procedures every 6 months. At the age of 6 years (2009), his respiratory parameters included a forced vital capacity (FVC) of $25 \%$ and a forced expiratory volume (FEV) of $25 \%$; later that same year, he was diagnosed with pulmonary hypertension $(90 \mathrm{mmHg}$ systolic, normal range $15-30 \mathrm{mmHg}$ ). He was treated with sildenafil to reduce his pulmonary hypertension in 2010 (7 years old), but at this stage, his respiratory status started to worsen. Initiation of bi-level positive 
airway pressure treatment increased the patient's wellbeing. In May and October of 2010, the left side and posterior thoracic rib cage were revised, respectively, with osteotomy of the posterior chest wall like the primary surgeries in 2004. At this time, the posterior and lateral chest walls were fully fused. At the age of 8 years, his respiratory status was rapidly deteriorating, and posterior revision surgery with resection of the posterior wall had no further positive influence on his respiratory function. The patient's physical condition worsened, and his respiratory status was even more characteristic of severe TIS. By this time, he could perform only short walks with increased strain, and the surgeons, parents and the patient did not foresee a benefit of further VEPTR surgery.

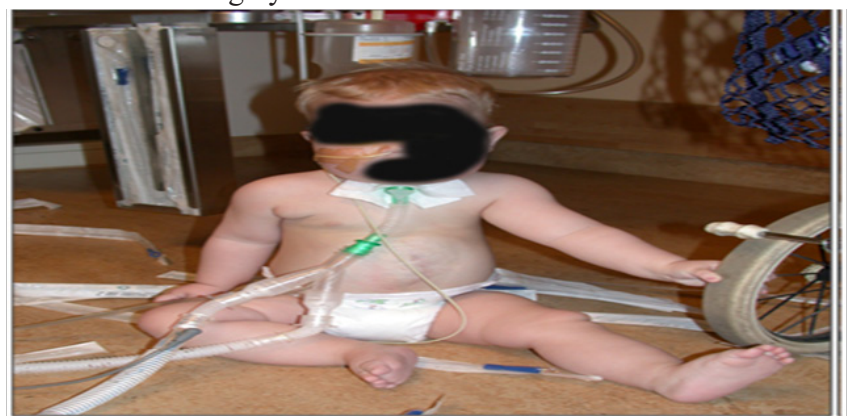

Figure I Image of the patient with a home ventilator before his initial VEPTR treatment.

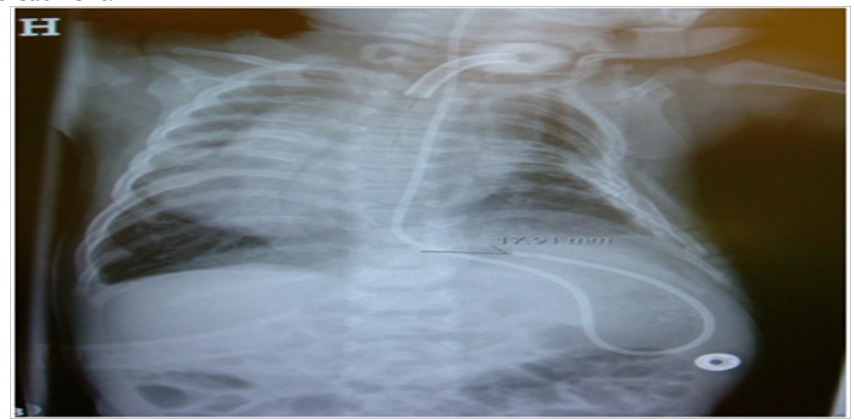

Figure 2 preoperative chest x-ray with short and local fused ribs.

\section{Surgical procedure}

Due to the lack of other options and the patient's rapid deterioration and life-threatening TIS, we agreed with the parents and the patient should undergo a sternal distraction procedure. To avoid problems with skin closure, the plastic surgeons applied a skin expander on the anterior parts of the left hemi-thorax several weeks before the procedure. In October 2011, an osteotomy of the sternum from the incisura jugularis to the processus xiphoideus was performed, and a 3-centimeter distraction of the sternum was achieved. The sternum was fixated with plates and screws (MXTGsternalFix5743F. DePuySynthes, Switzerland) (Figure 3). A structural autologous graft was used to fill the sternum diastasis (Figure 4).

After surgery, the patient experienced significant clinical improvement. His respiratory values stabilized, and his pulmonary artery values decreased to within the normal range. In November 2013, a second distraction of the sternum and an acute distraction were performed with the intention of further increasing the thoracic cage volume, both to optimize the patient's respiratory function and to accommodate his growth. During surgery, the fully integrated bone graft at the bone transplantation site was noted. The patient's sternum was again distracted 3 centimeters and was fixated with plates and screws (MXTGsternalFix5743F. DePuySynthes, Switzerland). The procedure was identical to the procedure performed in 2011, and the surgery and post-operative care were uneventful.

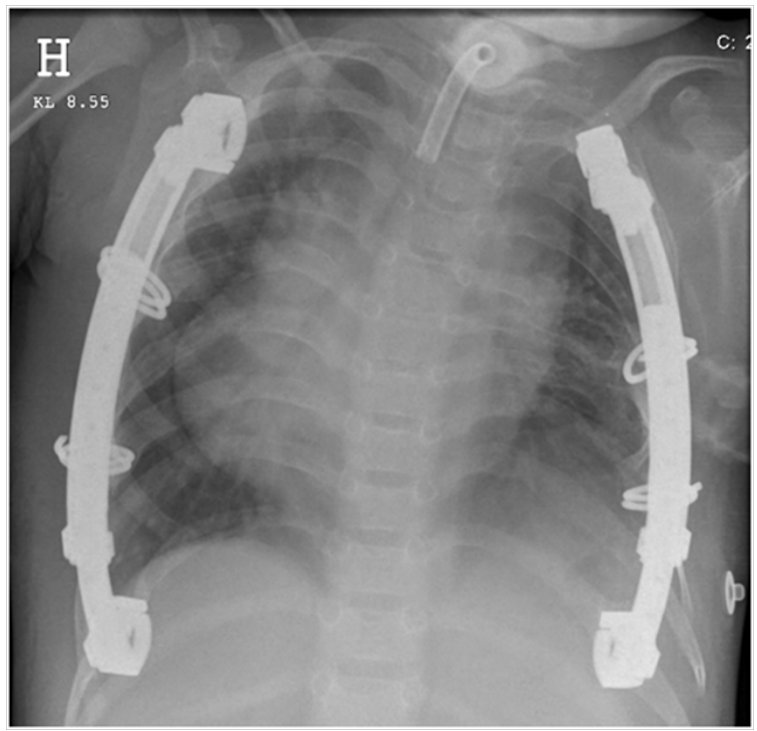

Figure 3 A. Chest X-ray showing the initial treatment with VEPTR.

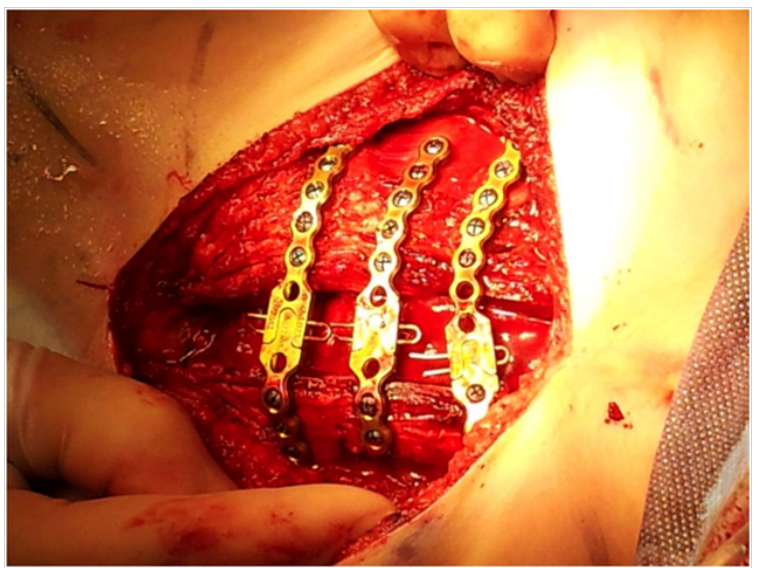

Figure 4 Per-operative image after sternotomy and plating.

\section{Discussion}

The treatment of severe SRS with TIS is controversial. Sternum distraction treatment in infancy has been previously reported in the literature, with partially poor results. ${ }^{5,8-11}$ and is associated with complications such as wound problems, lack of bone healing and subsequent lung parenchymal herniation. Our patient had a severe form of SRS with unilateral fused ribs. He was initially treated with bilateral VEPTR, opening wedge procedures and posterior rib resections. This treatment was successful for 5-6 years, but the patient started deteriorating at that time. Fusions of the ribs after VEPTR procedures have been documented and were found in our patient; this was likely the reason why the lengthening procedures failed to improve his thoracic volume. This finding is consistent with those of previous publications. ${ }^{12,13}$

The sternum distraction procedure was performed due to his poor ventilator status. Although this procedure is experimental and has the potential for major complications, no other options were available.

In their study, Ravitch et al. ${ }^{11}$ discussed the spectrum of asphyxiating thoracic dystrophy, a condition that resembles that of our patient; children with a mild form of the disease have good outcomes, as opposed to those with severe disease, who have poor prognoses. ${ }^{11}$ The only option for our patient's survival was to perform surgery that 
would allow for the expansion of his thoracic cage. Conroy et al. ${ }^{5}$ described an expansion of the thoracic cage by inserting distractors (MID System) between osteotomies of the ribs and slowly distracting the ribs, allowing callouses to form and eventually calcify. ${ }^{5}$ In this type of procedure, the patient must have a distractor device inserted through the skin during the distraction procedure for 10 days. In our case, we considered the risk of infection at a critical site to be too high to attempt this procedure. As in VEPTR surgery, we decided to perform an acute distraction and fixation. A structural allograft from our bone bank was used to fill the sternum gap, and the bone graft was fully integrated, which was verified at the second expansion procedure 2 years after the index distraction of the sternum. At least two other attempts to improve similar conditions have been described in the literature. The methods described included releasing several ribs at the costo-chondral junction and using a methyl methacrylate strut, but these attempts were unfortunately unsuccessful. ${ }^{79}$ Lateral thoracic expansion has also been described for the treatment of Jeune syndrome. ${ }^{8}$ but due to initial unilateral fused ribs and later bilateral posterior wall fusions, we concluded that the procedure was not well suited in our case.

TIS affects the lung function. Obtaining pulmonary respiratory values relies on patient cooperation, and reliable test values are most commonly registered after the age of 5 years. ${ }^{14}$ Our patient did not respond to VEPTR procedures with increased respiratory function, as measured by the FEV and FVC (Figure 6). The reason for this lack of response is not fully understood. Both values rely on wellfunctioning intercostal and accessory muscles (inspiration) and the diaphragm. Patients with numerous fused ribs primarily depend on a functional diaphragm for their respiration. Retrospectively, the lack of increased respiratory function measured by the FEV and FVC should have been expected. Although the respiratory values were relatively constant, the patient made a great clinical improvement. Before the first distraction of the sternum, he could speak 1-2 words between each breath and showed great fatigue after minimal physical strain. After surgery, he could speak full sentences, ride a bicycle to school and even participate in sports, such as cross-country skiing and soccer. The reason for this clinical improvement is poorly understood, but several non-respiratory parameters have been suggested as indirect measurements of respiratory improvement of restrictive lung disease. Parameters such as hemoglobin, weight and pulmonary arterial pressure have been introduced in recent reports as candidates for assessing pulmonary improvement following surgical lung expansion procedures.

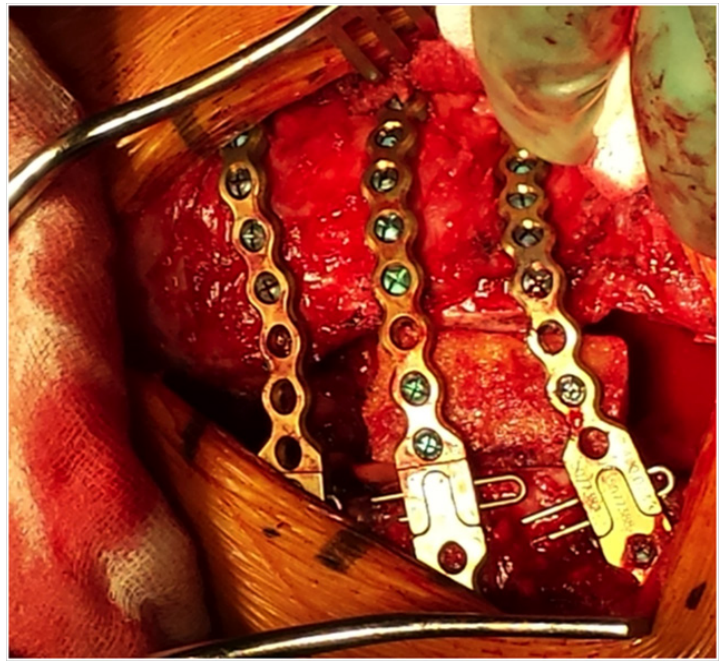

Figure 5 Per-operative image after the allograft was applied

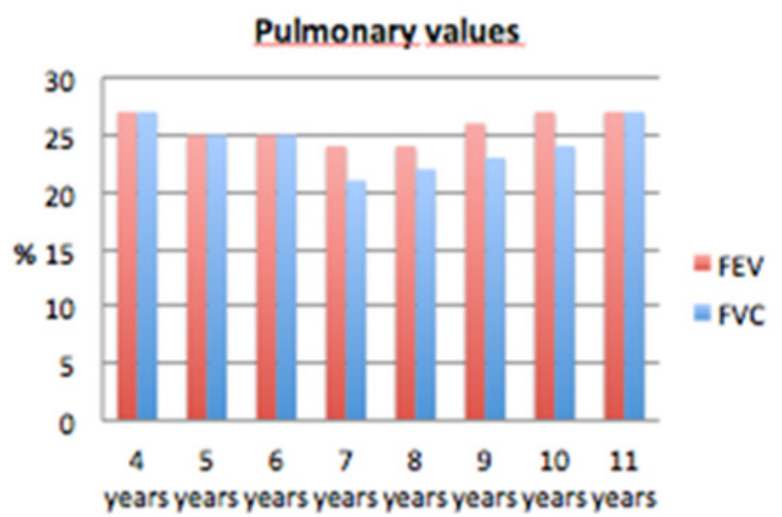

Figure 6 Graph showing forced expiratory volume (FEV) and forced vital capacity (FVC).

Secondary to the TIS, these patients often have pulmonary hypertension. Pulmonary hypertension was diagnosed in 2010 (age 7 years 5 months), and the patient was treated with sildenafil. At follow-up, after the first sternum distraction, the patient's values had normalized, and sildenafil was discontinued. Later, examinations have shown stable values within normal ranges.

According to Conroy et al. ${ }^{5}$ hemoglobin can be a valuable indicator for monitoring the effect of TIS treatment. Our patient did not respond to TIS with elevation of his hemoglobin levels. His values have remained stable at approximately $12.0 \mathrm{~g} / \mathrm{dl}$ from the first measurements in 2004 until today, with a peak of $13.8 \mathrm{~g} / \mathrm{dl}$ in 2014. The variation was indifferent to different surgical procedures. In a study by Caubet et al. ${ }^{15} 23 \%$ of patients with EOS (early onset scoliosis) showed signs of chronic hypoxia, and reports indicated that the patients' hemoglobin levels decreased significantly 6 to 24 months after VEPTR/opening wedge surgery. The greatest effect was observed in subjects with congenital scoliosis and rib fusion who underwent expansion thoracotomies with VEPTR implantation. ${ }^{15-17}$

Children with pulmonary insufficiency are often nutritionally deficient because their energy turnover due to the extra work of breathing is larger than their nutritional gains in eating. In severe respiratory insufficiency, even oral nutritional intake may represent a challenge because the patient may not tolerate the small breathing pause due to eating. ${ }^{18-20}$ Our patient was described as having major difficulties in gaining weight all his life and had suffered from a poor appetite for years due to abdominal pain immediately after oral food intake, which worsened his nutritional status. Even a feeding tube provoked abdominal pain. Recently, a possible gluten allergy was diagnosed, and since elimination of this protein (both oral and though the feeding tube), his abdominal pain has greatly diminished. However, he has failed to increase his weight ratio compared to his increase in height.

SRS is a disorder with a variety of respiratory problems, ranging from severe respiratory distress immediately after birth to relatively mild respiratory symptoms allowing normal growth and development. We successfully performed sternal distractions over a 3-year period in a juvenile boy. The patient had life-threatening TIS after an unsuccessful long course of VEPTR treatment. After distraction of the sternum, his clinical pulmonary status improved. To the best of our knowledge, these results have not been reported previously in this age group. We believe that this approach may offer an option for juveniles with severe TIS. 


\section{Patient consent form}

All written content and the use of all pictures have been approved by the patient and his family, who have given written informed consent. In our country, an approval from the National Ethics Committee is not needed for this type of report.

\section{Conflicts of interest}

The authors have no conflicts of interest to disclose.

\section{Acknowledgments}

None.

\section{References}

1. Burri PH Structural aspects of prenatal and postnatal development and growth of the lung. Lung growth and development. 1997;100:1-35.

2. Polgar G, Promadhat V Pulmonary function testing in children: techniques and standards. Saunders, Philadelphia, USA. 1971

3. Thurlbeck WM Postnatal human lung growth. Thorax. 1982;37(8):564-571.

4. Campbell RM, Smith MD, Mayes TC et al. The characteristics of thoracic insufficiency syndrome associated with fused ribs and congenital scoliosis. J Bone Joint Surg Am. 2003;85A(3):399-408.

5. Conroy E, Eustace N, McCormack D Sternoplasty and rib distraction in neonatal Jeune syndrome. J PediatrOrthop. 2010;30(6):527-530.

6. Aronson DC, Van Nierop JC, Taminiau A et al. Homologous bone graft for expansion thoracoplasty in Jeune's asphyxiating thoracic dystrophy. J Pediatr Surg. 1999;34(3): 500-503.

7. Barnes ND, Hull D, Milner AD, Waterston DJ Chest reconstruction in thoracic dystrophy. Arch Dis Child. 1971;46(250):833-837.

8. Davis JT, Heistein JB, Castile RG, Adler B, Mutabagani KH, et al. Lateral thoracic expansion for Jeune's syndrome: midterm results. Ann Thorac Surg. 2001;72(3):872-877.

9. Todd DW, Tinguely SJ, Norberg WJ A thoracic expansion technique for Jeune's asphyxiating thoracic dystrophy. $J$ Pediatr Surg. 1986;21(2):161-163.
10. Phillips D, van Aalst J Jeune's syndrome (asphyxiating thoracic dystrophy): congenital and acquired. Seminars in pediatric surgery. 2008;17(3):167-172.

11. Ravitch MW, Madden JJ Congenital Deformities of the Chest Wall and Their Operative Correction. Plastic and Reconstructive Surgery. 1979;63(1):117

12. Groenefeld B, Hell AK Ossifications after vertical expandable prosthetic titanium rib treatment in children with thoracic insufficiency syndrome and scoliosis. Spine (Phila Pa 1976). 2013;38(13):E819-E823.

13. Zivkovic V, Buchler P, Ovadia D, Riise R, Stuecker R, Extraspinal ossifications after implantation of vertical expandable prosthetic titanium ribs (VEPTRs). J Child Orthop. 2014;8(3):237-244.

14. England SJ Current techniques for assessing pulmonary function in the newborn and infant: advantages and limitations. Pediatr Pulmonol. $1988 ; 4(1): 48-53$.

15. Caubet JF, Emans JB, Smith JT et al. Increased hemoglobin levels in patients with early onset scoliosis: prevalence and effect of a treatment with Vertical Expandable Prosthetic Titanium Rib (VEPTR). Spine (Phila Pa 1976). 2009;34(23):2534-2536.

16. Midgren B, Petersson K, Hansson L et al. Nocturnal hypoxaemia in severe scoliosis. BrJ Dis Chest. 1988;82(3):226-236.

17. Bach JR, Robert D, Leger P, Langevin B Sleep fragmentation in kyphoscoliotic individuals with alveolar hypoventilation treated by NIPPV. Chest. 1995;107(6):1552-1558.

18. Atkinson SA Special nutritional needs of infants for prevention of and recovery from bronchopulmonary dysplasia. $J$ Nutr. 2001;131(3):942S-946S.

19. Abrams SA Chronic pulmonary insufficiency in children and its effects on growth and development. J Nutr. 2001;131(3):938S-9341S.

20. Skaggs DL, Sankar WN, Albrektson J et al. Weight gain following vertical expandable prosthetic titanium ribs surgery in children with thoracic insufficiency syndrome. Spine. 2009;34(23):2530-2533. 